\title{
SUMMATION AND UNCOUNTABLE BI-ORTHOGONAL SYSTEMS IN LOCALLY CONVEX SPACES
}

\author{
by HARRY F. JOINER, II \\ (Received 4th October 1972, revised 29th January 1973)
}

\section{Introduction}

The purpose of this paper is to extend to locally convex spaces and to uncountable systems several well-known results concerning infinite series, biorthogonal sequences, and Schauder bases. Section 2 gives three extensions of the theorem of Orlicz (10) and Pettis (11) and some lemmas that will be needed later. The third section introduces the notions of a summability basis and a summability basis of subspaces, and two main theorems are proved, including a simplification of Retherford and McArthur's proof (12) of a theorem of Nikol'skir (9). Section 4 investigates the positive cone of an uncountable biorthogonal system, particularly conditions equivalent to the regularity of this cone.

Throughout this paper, we adopt the following notation: $\mathrm{E}$ will denote a topological vector space and $E^{\prime}$ its topological dual. A locally convex space will be assumed Hausdorff. Let $A$ denote an arbitrary index set and $\mathscr{F}$ denote the set of all nonempty finite subsets of $A$.

\section{Summability and the Orlicz-Pettis Theorem}

Let $\left(x_{\alpha}\right)_{\alpha \in A}$ be an indexed family in $E$. The series, denoted by $\Sigma_{\alpha} x_{\alpha}$, is summable (unconditionally Cauchy) provided the net $\left(\Sigma_{a \in a} x_{\alpha}\right)_{a \in \mathscr{F}}$ converges (is Cauchy) in $E$ where $\mathscr{F}$ is directed by set inclusion. We write $\Sigma_{\alpha} x_{\alpha}=x$ if $x=\lim _{a} \Sigma_{\alpha \in a} x_{\alpha}$. For countable families $A$, summability of the series $\Sigma_{\alpha} x_{\alpha}$ is equivalent to unordered or unconditional convergence of the series. We shall need the following:

Lemma 1. In a topological vector space, the series $\Sigma_{\alpha} x_{\alpha}$ is unconditonally Cauchy if and only if for every countable sub-family $\left\{\alpha_{1}, \alpha_{2}, \ldots\right\}$ of $A$, the series $\sum_{i=1}^{\infty} x_{\alpha_{i}}$ is Cauchy.

Proof. Suppose that $\Sigma_{\alpha} x_{\alpha}$ is not unconditionally Cauchy. We now construct a non-Cauchy countable series. Let $U$ be a neighbourhood of $\theta$ such that for every $a \in \mathscr{F}$, there exists $b \in \mathscr{F}$ with $a \cap b=\varnothing$ and $\Sigma_{\alpha \in b} x_{\alpha} \notin U$. Let $a \in \mathscr{F}$, and choose $b_{1} \in \mathscr{F}$ such that $a \cap b_{1}=\varnothing$ and $\Sigma_{a \in b 1} x_{\alpha} \notin U$. Now choose $b_{2} \in \mathscr{F}$ such that $b_{1} \cap b_{2}=\varnothing$ and $\Sigma_{\alpha \in b_{2}} x_{\alpha} \notin U$. Similarly choose $b_{n} \in \mathscr{F}$ such that $b_{n} \cap\left(\bigcup_{1}^{n-1} b_{i}\right)=\varnothing$ and $\Sigma_{a \in b} x_{\alpha} \notin U$. Clearly, $B=\bigcup_{1}^{\infty} b_{n}$ is countable and $\Sigma_{\alpha \in B} x_{\alpha}$ is not Cauchy. The converse is obvious.

E.M.S. $-19 / 1-A$ 
The following lemma is due to Robertson (13, see his remark on p. 152 concerning Theorem 1).

Lemma 2. In a topological vector space, a series $\Sigma_{\alpha} x_{\alpha}$ is Cauchy if and only if the set $\left\{\Sigma_{a \in a} x_{\alpha}: a \in \mathscr{F}\right\}$ is precompact. Hence, the set of finite partial sums is bounded.

For locally convex spaces, we have the following extension by adapting McArthur's argument (8, Lemma 4):

Lemma 3. If $\Sigma_{\alpha} x_{\alpha}$ is a Cauchy series in a locally convex space $E$, then for each bounded set $B$ of the Banach space $m_{A}$ of bounded scalar-valued functions on $A$ with supremum norm, the set

\section{is precompact.}

$$
S(B)=\left\{\Sigma_{\alpha \in a} b_{\alpha} x_{\alpha}: b=\left(b_{\alpha}\right) \in B \text { and } a \in \mathscr{F}\right\}
$$

The following remarkable theorem was first proved in Banach spaces by Orlicz (10) and Pettis (11) and extended to the following form by Grothendieck (2), McArthur (6) and Robertson (13):

Theorem. (Orlicz-Pettis) In a locally convex space $E$, each subseries of a (countable) series converges with respect to the initial topology on $E$ if and only if each subseries of the series converges with respect to the $\sigma\left(E, E^{\prime}\right)$ topology.

For countable series, subseries convergence implies unconditional convergence (summability), and for sequentially complete spaces, the two notions are equivalent.

Theorem 1. If $E$ is a complete locally convex space, then $\Sigma_{\alpha} x_{\alpha}$ is summable whenever every countable subseries $\sum_{i=1}^{\infty} x_{\alpha_{i}}$ is $\sigma\left(E, E^{\prime}\right)$-convergent.

Proof. This is immediate from the completeness since Robertson's proof (13) of the Orlicz-Pettis Theorem implies the series is unconditionally Cauchy, although not necessarily summable as the following example illustrates.

Let $E$ be the subspace of all countably nonzero elements in an uncountable product $\prod_{\alpha \in A} \mathbf{R}$ of reals with the product topology. Let $e_{\alpha}$ be the element of $E$ with 1 as the $\alpha$ coordinate and 0 elsewhere. Then every countable subfamily $\left(e_{\alpha_{i}}\right)_{i=1}^{\infty}$ sums to the element with 1 at $\alpha_{i}(i=1,2, \ldots)$ and 0 elsewhere, but the whole family sums to the element of $\prod_{\alpha \in A} R$ with 1 in every coordinate, which, of course, is not in $E$.

Theorem 2. If $E$ is a $\sigma\left(E, E^{\prime}\right)$-sequentially complete locally convex space then $\Sigma_{\alpha} x_{\alpha}$ is strongly summable if and only if $\Sigma_{\alpha} x_{\alpha}$ is $\sigma\left(E, E^{\prime}\right)$-summable.

Proof. It is necessary only to show that $\Sigma_{\alpha} x_{\alpha}$ is strongly summable when it is $\sigma\left(E, E^{\prime}\right)$-summable. If $\Sigma_{\alpha} x_{\alpha}$ is $\sigma\left(E, E^{\prime}\right)$-summable to $x$, then every countable subseries $\sum_{i=1}^{\infty} x_{\alpha_{i}}$ is $\sigma\left(E, E^{\prime}\right)$-Cauchy by Lemma 1 . The sequential completeness of $E$ implies that $\sum_{i=1}^{\infty} x_{x_{i}} \sigma\left(E, E^{\prime}\right)$-con verges, and hence strongly converges 
by the Orlicz-Pettis Theorem. From Lemma 1, we conclude that the series $\Sigma_{\alpha} x_{\alpha}$ is Cauchy in the original topology of $E$. Since $\Sigma_{\alpha} x_{\alpha}$ is $\sigma\left(E, E^{\prime}\right)$-summable to $x$ and strongly Cauchy, $\Sigma_{\alpha} x_{\alpha}$ is strongly summable to $x$.

A third variation is

Theorem 3. Let $E$ be a locally convex space. If $\Sigma_{\alpha} x_{\alpha}$ is $\sigma\left(E, E^{\prime}\right)$-summable and every countable subseries $\sum_{i=1}^{\infty} x_{\alpha_{l}} \sigma\left(E, E^{\prime}\right)$-converges, then $\Sigma_{\alpha} x_{\alpha}$ is strongly summable.

The proof is straightforward and will be omitted.

Remark. For uncountable index sets, the Orlicz-Pettis Theorem may be proved by applying Theorem 3 (which requires only the countable form of the Orlicz-Pettis Theorem in its proof).

In a locally convex space, every subseries of a series is summable if and only if every subseries is $\sigma\left(E, E^{\prime}\right)$-summable.

\section{Summability bases}

In this section we shall state and prove the theorems in very general terms and restate them in more useful and concrete terms as corollaries. The following definitions are extensions of the well-known concepts for Banach spaces of a Schauder basis and a Schauder basis of subspaces (because of summability we must restrict our attention essentially to unconditional bases). The system $\left(x_{\alpha} ; f_{\alpha}\right)_{\alpha \in A}$ is bi-orthogonal provided $\left\{x_{\alpha}: \alpha \in A\right\} \subset E$ and $\left\{f_{\alpha}: \alpha \in A\right\} \subset E^{\prime}$ and $f_{a}\left(x_{\beta}\right)=\delta_{\alpha \beta}$, the Kronecker symbol. An (unconditional) Schauder basis for $E$ is a countable bi-orthogonal system $\left(x_{n} ; f_{n}\right)$ such that $\sum_{n=1}^{\infty} f_{n}(x) x_{n}$ (unconditionally) converges to $x$ for every $x \in E$. A bi-orthogonal system $\left(x_{\alpha} ; f_{\alpha}\right)_{\alpha \in A}$ is a summability basis for $E$ provided $\Sigma_{\alpha} f_{\alpha}(x) x_{\alpha}$ is summable to $x$ for each $x \in E$. (For countable systems, a summability basis is an unconditional Schauder basis and vice versa.) For a bi-orthogonal system $\left(x_{\alpha} ; f_{\alpha}\right)_{a \in A}$, define

$$
S_{a}(x)=\Sigma_{\alpha \in a} f_{\alpha}(x) x_{\alpha}
$$

for each $x \in E$ and each $a \in \mathscr{F}$. Then $S_{a}$ is the a-partial sum operator of $\left(x_{\alpha} ; f_{\alpha}\right)$. A summability basis $\left(x_{\alpha} ; f_{\alpha}\right)$ is equicontinuous if $\left\{S_{a}: a \in \mathscr{F}\right\}$ is an equicontinuous family of operators on $E$. Summability bases have received recent attention by Knowles (4) and Marti (5).

Let $\left(E_{\alpha}\right)_{\alpha \in A}$ be a family of subspaces of $E$ such that there is a unique family $\left(P_{\alpha}\right)_{\alpha \in A}$ of continuous projections on $E$ with $P_{\alpha}(E)=E_{\alpha}$ and $P_{\alpha} P_{\beta}=0$ for $\alpha \neq \beta$ and $\Sigma_{\alpha} P_{\alpha}(x)$ is summable to $x$ for each $x \in E$. Then $\left(E_{\alpha} ; P_{\alpha}\right)$ is a summability basis of subspaces for $E$. The a-partial sum operator for $\left(E_{\alpha} ; P_{\alpha}\right)$ is $S_{\alpha}=\Sigma_{\alpha \in a} P_{\alpha}$ for each $a \in \mathscr{F}$. The basis $\left(E_{\alpha} ; P_{\alpha}\right)_{\alpha \in A}$ is equicontinuous if $\left\{S_{a}: a \in \mathscr{F}\right\}$ is an equicontinuous family of operators on $E$. Every summability basis $\left(x_{\alpha} ; f_{\alpha}\right)_{\alpha \in A}$ corresponds obviously to the summability basis of subspaces $\left(E_{a} ; P_{\alpha}\right)_{\alpha \in A}$ where $E_{\alpha}=\operatorname{span}\left(x_{\alpha}\right)$ and $P_{\alpha}(x)=f_{\alpha}(x) x_{\alpha}$ for each $x$ and each $\alpha$. 
Remark. A summability basis in a barrelled space is necessarily equicontinuous (by Lemma 2 and (14, Theorem 3, p. 69)).

We shall use the following lemma whose proof is indicated since we could find no direct reference.

Lemma 4. Let $E$ and $F$ be topological vector spaces with $F$ complete. Let $S$ be a family of continuous linear functions from $E$ into $F$ and let $S^{*}$ denote the family of continuous extensions of the functions in $S$ to the completion $E^{*}$ of $E$. Then $S$ is equicontinuous if and only if $S^{*}$ is equicontinuous.

Proof. This is clear since the closed neighbourhoods of $E^{*}$ are exactly the closures (in $E^{*}$ ) of neighbourhoods in $E(1$, p. 62) and the closed neighbourhoods of $F$ form a neighbourhood base.

One common example of a summability basis of subspaces occurs in the product $E$ of the spaces $\left\{E_{\alpha}: \alpha \in A\right\}$; for, if $P_{\alpha}$ is the canonical projection of $E$ on to $E_{\alpha}$ and if $E$ has the product topology, then $\left(E_{\alpha} ; P_{\alpha}\right)_{\alpha \in A}$ is a summability basis of subspaces. This will be an equicontinuous basis when the spaces $E_{\alpha}(\alpha \in A)$ are barrelled since $E$ will be barrelled (14, p. 94).

The equicontinuity condition is important because it allows us to extend the basis, if necessary, to the completion.

Theorem 4. Let $E$ be a Hausdorff topological vector space and let $\left(E_{a}\right)_{a \in A}$ be a family of complete subspaces with $M=\operatorname{span}\left(\cup_{a \in A} E_{\alpha}\right)$ and $M^{\prime}$, the closure of $M$ in $E$. If there exist a family of continuous projections $\left(P_{\alpha}\right)_{\alpha \in A}$ on $M$ such that $\left(E_{\alpha} ; P_{\alpha}\right)_{\alpha \in A}$ is an equicontinuous summability basis of subspaces for $M$, and if $P_{\alpha}^{\prime}(\alpha \in A)$ is the continuous extension of $P_{\alpha}$ to $M^{\prime}$ and $S_{a}^{\prime}=\Sigma_{z \in a} P_{\alpha}^{\prime}(a \in \mathscr{F})$, then $\left(E_{\alpha} ; P_{\alpha}^{\prime}\right)$ is an equicontinuous summability basis for $M^{\prime}$.

Proof. Clearly, the image of $P_{\alpha}^{\prime}$ is $E_{\alpha} ; P_{\alpha}^{\prime}$ is a continuous projection; and $P_{\alpha}^{\prime} P_{\beta}^{\prime}=0$ for $\alpha \neq \beta$. Let $x \in M^{\prime}$. We complete the proof by showing that

$$
\lim _{a} S_{a}^{\prime}(x)=x
$$

Let $V$ be a neighbourhood of $\theta$ in $M^{\prime}$. There is a balanced neighbourhood $U$ of $\theta$ such that $U+U+U \subset V$. Since $\left\{S_{a}^{\prime}: a \in \mathscr{F}\right\}$ is equicontinuous by Lemma 4, there is a balanced neighbourhood $W$ of $\theta$ such that $S_{a}^{\prime}(W) \subset U$ for all $a \in \mathscr{F}$. Now there is $y \in M$ such that $y-x \in W \cap U$. Hence,

$$
S_{a}^{\prime}(y-x)=S_{a}^{\prime}(y)-S_{a}^{\prime}(x) \in U
$$

for every $a \in \mathscr{F}$. Since $\left(E_{\alpha}, P_{a}\right)$ is a summability basis for $M$, there exists $a \in \mathscr{F}$ such that

$$
y-S_{b}^{\prime}(y)=y-S_{b}(y) \in U
$$

for every $b \in \mathscr{F}$ with $a \subset b$. Let $a \subset b \in \mathscr{F}$. Then

$$
x-S_{b}^{\prime}(x)=(x-y)+\left(y-S_{b}^{\prime}(y)\right)+\left(S_{b}^{\prime}(y)-S_{b}^{\prime}(x)\right) \in U+U+U \subset V .
$$


Thus, $\lim _{a} S_{a}^{\prime}(x)=x$. for every $x \in M^{\prime}$.

We have the following obvious corollaries:

Corollary 4.1. Let $E$ be a Hausdorff topological vector space and let $\left(x_{\alpha} ; f_{a}\right)_{\alpha \in A}$ be a bi-orthogonal system which is a summability basis for the span $M$ of $\left(x_{\alpha}\right)_{\alpha \in A}$. If $S_{a}(x)=\Sigma_{\alpha \in a} f_{\alpha}(x) x_{\alpha}$ for $x \in E$ and $a \in \mathscr{F}$ and if $\left\{S_{a}: a \in \mathscr{F}\right\}$ is equicontinuous, then $\left(x_{\alpha} ; f_{\alpha}\right)$ is a summability basis for $M^{\prime}$, the closure of $M$.

Corollary 4.2. Let $E$ be a Hausdorff topological vector space and let $\left(x_{n} ; f_{n}\right)$ be an equicontinuous Schauder basis for the span $M$ of $\left(x_{n}\right)$. Then $\left(x_{n} ; f_{n}\right)$ is an equicontinuous Schauder basis for M-closure.

The following theorem (7, Theorem 1) is due to McArthur and is stated here for the sake of completeness.

Theorem (McArthur). Let $\left\{S_{i}\right\}_{i \in I}$ be a family of linear functions from a vector space $E$ into a topological vector space $(F, \mathscr{T})$ and suppose that

$$
\left\{S_{i}(x): i \in I\right\}
$$

is bounded for each $x \in E$. Then there exists a weakest vector topology $\mathscr{T}^{\prime}$ for $E$ such that $\left\{S_{i}\right\}_{i \in I}$ is $\mathscr{T}^{\prime}-\mathscr{T}$ equicontinuous. If $\mathscr{T}$ is locally convex, then so is $\mathscr{T}^{\prime}$. If $(F, \mathscr{T})$ is Hausdorff, then $\left(E, \mathscr{T}^{\prime}\right)$ is Hausdorff if and only if $S_{i}(x)=\theta$ for all $i \in I$ implies $x=\theta$. If $(F, \mathscr{T})$ is locally convex and $\mathscr{T}$ is generated by a family $\Gamma=\{p\}$ of seminorms then $\mathscr{T}^{\prime}$ is generated by the family $\Gamma^{\prime}=\left\{p^{\prime}\right\}$ of seminorms where for each $p \in \Gamma, p^{\prime}(x)=\sup _{i \in I} p\left(S_{i}(x)\right)$.

The topology $\mathscr{T}^{\prime}$ defined in McArthur's Theorem is called the projective equicontinuous topology on $E$.

The following extends the theorem of Nikol'skii (9).

Theorem 5. Let $(E, \mathscr{T})$ be a locally convex space and let $\left(E_{\alpha}\right)_{\alpha \in A}$ be a family of complete subspaces of $E$ such that the span of $\cup_{\alpha \in A} E_{\alpha}$ is dense in $E$ and $E_{\alpha} \cap E_{\beta}=\{\theta\}$ for all $\alpha \neq \beta$. Let $\Gamma$ be a family of seminorms generating the topology $\mathscr{T}$. If for each $p \in \Gamma$, there exist $q \in \Gamma$ and a positive constant $K=K(p)$ such that

$$
p\left(\Sigma_{\alpha \in a} x_{z}\right) \leqq K q\left(\Sigma_{\alpha \in b} x_{\alpha}\right)
$$

for arbitrary $a, b \in \mathscr{F}$ with $a \subset b$ and arbitrary $x_{\alpha} \in E_{\alpha}(\alpha \in b)$, then $\left(E_{\alpha}\right)$ is $a$ summability basis of subspaces for $E$.

Proof. Let $M$ be the span of $\cup_{\alpha} E_{\alpha}$. If $\Sigma_{\alpha} x_{\alpha}=\theta$ and $x_{\alpha} \in E_{\alpha}$, then for $p \in \Gamma$ and fixed $\alpha_{0} \in A$, we have $p\left(x_{\alpha_{0}}\right) \leqq 2 K q\left(\Sigma_{\alpha \in a} x_{\alpha}\right)$ for all $a \in \mathscr{F}$ with $\alpha_{0} \in a$. Since $E$ is Hausdorff and the right-hand side converges to 0 on $\mathscr{F}$, we have $x_{\alpha_{0}}=\theta$. Define $P_{\alpha}(x)=x_{\alpha}$ for $x \in M$ and $\alpha \in A$. For $a \in \mathscr{F}$, let $S_{a}=\Sigma_{\alpha \in a} P_{\alpha}$. Let $\mathscr{T}^{\prime}$ be the projective equicontinuous topology on $M$ defined as above using the family $\left\{S_{a}: a \in \mathscr{F}\right\}$ and the topology $\mathscr{T}$ on $E$. Then $\left\{S_{a}(x): a \in \mathscr{F}\right\}$ and finite, and hence bounded, for each $x \in M$; and we may apply McArthur's Theorem. Thus, $\mathscr{T}^{\prime}$ is defined by the family $\Gamma^{\prime}=\left\{p^{\prime}: p \in \Gamma\right\}$ of seminorms 
where $p^{\prime}(x)=\sup _{a \in \mathscr{F}} p\left(\Sigma_{\alpha \in a} x_{a}\right)$ for each $x \in M$ and $p \in \Gamma$; and $\left\{S_{a}: a \in \mathscr{F}\right\}$ is $\mathscr{T}^{\prime}-\mathscr{T}$ equicontinuous. It is easy to see that (1) implies $p^{\prime}(x) \leqq K q(x)$, and of course, $p(x) \leqq p^{\prime}(x)$ for all $x \in M$ and $p \in \Gamma$. Hence, $\mathscr{T}^{\prime}$ is equivalent to the topology induced on $M$ by $\mathscr{T}$ and $\left\{S_{a}\right\}$ is equicontinuous for $\mathscr{T}$. Thus $\left(E_{\alpha} ; P_{\alpha}\right)$ is an equicontinuous summability basis for $M$. By Theorem 4, $\left(E_{\alpha} ; P_{\alpha}^{\prime}\right)$ is an equicontinuous summability basis for $E$, the closure of $M$.

Corollary 5.1. Let $(E, \mathscr{T})$ be a locally convex space and let $\left(x_{\alpha}\right)_{\alpha \in A}$ be a family in $E$ with $x_{\alpha} \neq \theta$ and the span of $\left(x_{\alpha}\right)$ dense in $E$. Let $\Gamma$ be a family of seminorms generating the topology $\mathscr{T}$. If for each $p \in \Gamma$, there exist $q \in \Gamma$ and $a$ positive constant $K=K(p)$ such that

$$
p\left(\Sigma_{\alpha \in a} \lambda_{\alpha} x_{\alpha}\right) \leqq K q\left(\Sigma_{\alpha \in b} \lambda_{\alpha} x_{\alpha}\right)
$$

for arbitrary scalars $\lambda_{\alpha}(\alpha \in b)$ and for arbitrary $a, b \in \mathscr{F}$ with $a \subset b$, then $\left(x_{\alpha}\right)_{\alpha \in A}$ is a summability basis for $E$.

The next corollary removes the completeness requirement in the theorem (12, Theorem 3.1) of Retherford and McArthur for locally convex spaces.

Corollary 5.2. Let $(E, \mathscr{T})$ be a locally convex space and let $\left(x_{n}\right)_{n=1}^{\infty}$ be a sequence in $E$ with $x_{n} \neq \theta$ for all $n$ and the span of $\left(x_{n}\right)$ dense in $E$. Let $\Gamma$ be a family of seminorms generating the topology $\mathscr{T}$. If for each $p \in \Gamma$, there exist $q \in \Gamma$ and a positive constant $K=K(p)$ such that

$$
p\left(\sum_{n=1}^{k} \lambda_{n} x_{n}\right) \leqq K q\left(\sum_{n=1}^{k+m} \lambda_{n} x_{n}\right)
$$

for arbitrary scalars $\left(\lambda_{n}\right)$ and positive integers $k$ and $m$, then $\left(x_{n}\right)$ is a Schauder basis for $E$.

Proof. Note that the basis in this case may be a conditional Schauder basis. The above proof is modified by using the family $\left\{S_{n}\right\}$ of operators defined by

$$
S_{n}\left(\sum_{i=1}^{k} \lambda_{i} x_{i}\right)=\left\{\begin{array}{l}
\sum_{i=1}^{n} \lambda_{i} x_{i}, \text { for } n \leqq k, \\
\sum_{i=1}^{k} \lambda_{i} x_{i}, \text { for } k<n,
\end{array}\right.
$$

instead of the unordered partial sums $\left\{S_{a}\right\}$.

A corresponding result for a basis of complete subspaces also holds.

Since condition (1) of Theorem 5 is essentially the equicontinuity of the finite partial sum operators, we have the following partial converse which includes all Banach, Fréchet, and reflexive spaces:

Theorem 6. If $E$ is barrelled and has a summability basis (of subspaces), then condition (1) holds. 


\section{Positive cones of bi-orthogonal systems}

Let $E$ be a real vector space. A set $K$ in $E$ is a cone provided:

(i) $K+K \subset K$,

(ii) $\lambda K \subset K$ for all $\lambda \geqq 0$, and

(iii) $K \cap(-K)=\{\theta\}$.

A set satisfying (i) and (ii) is called a wedge. A cone $K$ in $E$ induces a partial ordering $\leqq$ on $E$ by $x \leqq y$ if and only if $y-x \in K$. If $E$ is ordered in this way by a cone, we call $E$ an ordered vector space. Clearly, $K=\{x: \theta \leqq x\}$.

Let $\left(x_{\alpha} ; f_{\alpha}\right)_{\alpha \in A}$ be a bi-orthogonal system on $E$. Let

$$
K=\left\{x \in E: f_{\alpha}(x) \geqq 0 \text { for all } \alpha \in A\right\} .
$$

Clearly, $K$ is a wedge in $E$ and $K$ will be a cone if and only if $\left\{f_{\alpha}:{ }_{\alpha \in A}\right\}$ is total over $E$, i.e. $f_{\alpha}(x)=0$ for $\alpha \in A$ implies $x=\theta$. We call $K$ the positive cone of the bi-orthogonal system $\left(x_{\alpha} ; f_{a}\right)$ (when $\left\{f_{a}\right\}$ is total).

Let $E$ be ordered by the cone $K$, and let $[x, y]$ denote the order interval

$$
\{z: x \leqq z \leqq y\}=(x+K) \cap(y-K) \text {. }
$$

Then $K$ is regular (sequentially regular) provided every increasing net (sequence) in $K$ which is contained in an order interval converges to an element of $K$. The following is an extension to general bi-orthogonal systems of a recent theorem of McArthur (8).

Theorem 7. Let $E$ be a locally convex space and $\left(x_{\alpha} ; f_{\alpha}\right)_{\alpha \in A}$ a bi-orthogonal system on $E$ with $\left\{f_{\alpha}\right\}$ total over $E$. Let $E$ be ordered by the positive cone $K$ of $\left(x_{\alpha} ; f_{\alpha}\right)$. The following statements are equivalent:

(i) For each $x \in K$ and each bounded non-negative real-valued function $\left(\lambda_{\alpha}\right)_{\alpha \in A}$, the series $\Sigma_{\alpha} \lambda_{\alpha} f_{\alpha}(x) x_{\alpha}$ is summable,

(ii) $[\theta, x]$ is compact for each $x \in K$,

(iii) $[\theta, x]$ is weakly compact for each $x \in K$,

(iv) $K$ is weakly regular,

(v) $K$ is regular,

(vi) $[\theta, x]$ is complete, $\sigma\left(E, E^{\prime}\right)$-sequentially complete and bounded for each $x \in K$.

The above conditions imply the following condition and are equivalent to it if $\Sigma_{\alpha} f_{\alpha}(x) x_{\alpha}$ converges for every $x \in K$ :

(vii) The supremum of each order bounded subset of $K$ exists and is an element of $K$.

If $K$ is complete, then (i)-(vi) are also equivalent to the following statements:

(viii) $K$ is sequentially regular,

(ix) $K$ is weakly sequentially regular. 
Proof. With obvious modifications, based on Lemma 3 for (i) $\Rightarrow$ (ii) McArthur's proof extends for the following implications:

(i) $\Rightarrow$ (ii) $\Rightarrow($ (iii) $\Rightarrow$ (iv) $\Rightarrow($ ix $) \Leftrightarrow$ (viii) and (ii) $\Rightarrow(v) \Rightarrow($ i $) \Rightarrow$ (vii) and (v) $\Rightarrow$ (iv), and clearly, ((ii) and (iii)) $\Rightarrow$ (vi). Thus, to complete the proof we need only show that (iv) $\Rightarrow(\mathrm{i})$, (vi) $\Rightarrow(\mathrm{i})$, (viii) $\Rightarrow$ (i) (if $K$ is complete) and (vii) $\Rightarrow$ (i) (if $\Sigma_{\alpha} f_{\alpha}(x) x_{\alpha}$ is summable for $x \in K)$. Let $\left(\lambda_{\alpha}\right)_{\alpha \in A}$ be a bounded non-negative real-valued function on $A$ and $x \in K$. In each case we must show that $\Sigma_{\alpha} \lambda_{\alpha} f_{\alpha}(x) x_{\alpha}$ is summable.

Suppose (iv) holds. If $\lambda=\sup _{\alpha} \lambda_{\alpha}$, then $\theta \leqq \Sigma_{\alpha \in a} \lambda_{\alpha} f_{\alpha}(x) x_{\alpha} \leqq \lambda x$ for all $a \in \mathscr{F}$. In addition, if $a \subset b$ and $a, b \in \mathscr{F}$, then $\Sigma_{\alpha \in a} \lambda_{\alpha} f_{\alpha}(x) x_{\alpha} \leqq \Sigma_{\alpha \in b} \lambda_{\alpha} f_{\alpha}(x) x_{\alpha}$. Hence, $\Sigma_{\alpha} \lambda_{\alpha} f_{\alpha}(x) x_{\alpha}$ and every subseries of it are $\sigma\left(E, E^{\prime}\right)$-summable by (iv). By Theorem $3, \Sigma_{\alpha} \lambda_{\alpha} f_{\alpha}(x) x_{\alpha}$ is strongly summable.

Assume (vi) holds. Then $\left\{\Sigma_{\alpha \in a} \lambda_{\alpha} f_{\alpha}(x) x_{\alpha}: a \in \mathscr{F}\right\}$ is bounded since it is a subset of $[\theta, \lambda x]$, which is bounded. Hence, for each

$$
f \in E^{\prime}, \Sigma_{\alpha}\left|\lambda_{a} f_{a}(x) f\left(x_{\alpha}\right)\right|<+\infty .
$$

It follows that each countable subseries is $\sigma\left(E, E^{\prime}\right)$-Cauchy and by hypothesis, $\sigma\left(E, E^{\prime}\right)$-convergent. Hence, as in the proof of Theorem $2, \Sigma_{\alpha} \lambda_{\alpha} f_{\alpha}(x) x_{\alpha}$ is strongly Cauchy and by the assumed completeness of $[\theta, \lambda x]$, is summable.

Next, assume $K$ is complete and sequentially regular. Then every countable subseries $\sum_{i=1}^{\infty} \lambda_{\alpha_{i}} f_{\alpha_{i}}(x) x_{\alpha_{i}}$ is increasing and bounded above by $\lambda x$, and hence convergent. Now by Lemma 1 , the series $\Sigma_{\alpha} \lambda_{\alpha} f_{\alpha}(x) x_{\alpha}$ is Cauchy and by the completeness of $K$, is summable.

We conclude the proof of the theorem by noting that McArthur's proof for (vii) $\Rightarrow$ (i) (our numbers) requires the added hypothesis that $\Sigma_{\alpha} f_{\alpha}(x) x_{\alpha}$ converge for all $x \in K$ (even in the countable case) and that this is sufficient by the extension of (8, Lemma 5). Consider the space $m$ of bounded sequences with the bi-orthogonal sequence $\left(e_{n} ; f_{n}\right)$ where $e_{n}$ is the sequence with 1 in the $n$th coordinate and 0 elsewhere. Then $K$ is the usual positive cone of $m$ which has property (vii). The sequence $\left(\sum_{n=1}^{k} e_{n}\right)_{k}$ (determined by $(1) \in K$ ) does not converge in $m$ (contradicting (i)).

The order interval $[\theta, x]$ will be metrisable if and only if $\left\{\alpha ; f_{a}(x) \neq 0\right\}$ is countable (3).

Corollary 7.1. Let $E$ be a locally convex space, $\left(x_{\alpha} ; f_{\alpha}\right)_{\alpha \in A}$ a bi-orthogonal system on $E$ with $\left\{f_{\alpha}\right\}$ total over $E$, and $K$ the positive cone of $\left(x_{\alpha} ; f_{\alpha}\right)$. If $K$ is regular and $E=K-K$, then $\left(x_{\alpha} ; f_{\alpha}\right)$ is a summability basis for $E$. If $K$ is complete, the converse is true.

Proof. For the first part, we need only show that $\Sigma_{\alpha} f_{\alpha}(x) x_{\alpha}$ is summable to $x$ for every $x \in E$. Since $E=K-K$, if suffices to consider only $x \in K$. Let $x \in K$. By (i) in Theorem 7, $\Sigma_{\alpha} f_{\alpha}(x) x_{\alpha}$ is summable. By the bi-orthogonality and totality of $\left\{f_{a}\right\}$, the sum must be $x$. Conversely, suppose that $K$ is complete and $\left(x_{\alpha} ; f_{a}\right)$ 


\section{BI-ORTHOGONAL SYSTEMS IN LOCALLY CONVEX SPACES · 9}

is a summability basis. Let $x \in K$ and $\left(\lambda_{a}\right)_{\alpha \in A}$ be a bounded non-negative realvalued function on $A$. Now $\Sigma_{\alpha} f_{\alpha}(x) x_{\alpha}$ is summable, and thus, by Lemma 3 , $\left\{\Sigma_{a \in a} \lambda_{a} f_{a}(x) x_{a}: a \in \mathscr{F}\right\}$ is precompact. Lemma 2 and the completeness of $K$ imply that $\Sigma_{\alpha} \lambda_{\alpha} f_{\alpha}(x) x_{\alpha}$ is summable. Hence, condition (i) of Theorem 7 is satisfied and $K$ is regular. Now let $x \in E$. Let $B=\left\{\alpha \in A: f_{\alpha}(x) \geqq 0\right\}$ and $C=\left\{\alpha \in A: f_{\alpha}(x)<0\right\}$. By condition (i) also, $\Sigma_{\alpha \in B} f_{\alpha}(x) x_{\alpha}$ is summable to, say, $y$ and $\Sigma_{\alpha \in C} f_{\alpha}(x) x_{\alpha}$ is summable to, say, $-z$. Clearly, $y \in K$ and $z \in K$ and $x=y-z$. Hence, $K-K=E$.

We now consider two examples. Let $A$ be uncountable and consider the product $\Pi_{\alpha: A} \mathbf{R}$ of reals with the product topology. Let $E$ be the countably non-zero elements and $\left(e_{\alpha} ; f_{\alpha}\right)_{\alpha \in A}$ the "unit vector" basis mentioned earlier. The positive cone of $\left(e_{\alpha} ; f_{\alpha}\right)$ is not complete but is regular (by condition (i) of Theorem 7). Let $F$ represent the countable nonconstant elements of the product. The system $\left(e_{\alpha} ; f_{\alpha}\right)$ is again a summability basis, but the positive cone is not complete and not regular (because condition (i) fails), but is sequentially regular (since the coordinatewise supremum is in $F$ and is clearly the limit of an increasing sequence).

\section{REFERENCES}

(1) R. E. Edwards, Functional Analysis, (Holt, Rinehart and Winston, New York, 1965).

(2) A. Grothendick, Sur les applications linéaires faiblement compactes d'espaces du type C(K), Canad. J. Math. 5 (1953), 129-172.

(3) H. F. JoINER, II, and T. A. CooK, Properties of summability bases and absolute summability bases in locally convex spaces, submitted for publication.

(4) R. J. KNOWLES, Schauder bases, bounded finiteness, and summability bases in locally convex spaces, dissertation, Univ. of Mass., Amherst (1972).

(5) J. T. MarTI, Extended bases for Banach spaces, Illinois J. Math. 15 (1971), 135-143.

(6) C. W. McArthur, On a theorem of Orlicz and Pettis, Pacific J. Math. 22 (1967), 297-302.

(7) C. W. McArthur, The projective equicontinuous topology, Proceedings of the Conference on Projections and Related Topics (Clemson Univ., Clemson, S. Carolina, 1968).

(8) C. W. MCARThuR, Convergence of monotone nets in ordered topological vector spaces, Studia Math. 34 (1970), 1-16.

(9) V. N. NiKol'sKiII, The best approximation and a basis in Fréchet space, Dokl. Akad. Nauk. SSSR 59 (1948), 639-642.

(10) W. Orlicz, Beiträge zur Theorie der Orthogonalentwicklungen II, Studia Math. 1 (1929), 241-255. 
(11) B. J. Petris, On integration in vector spaces, Trans. Amer. Math. Soc. 44, (1938), 277-304.

(12) J. R. REThERford and C. W. MCARTHUR, Some remarks on bases in linear topological spaces, Math. Ann. 164 (1966), 38-41.

(13) A. P. Robertson, On unconditional convergence in topological vector spaces, Proc. Roy. Soc. Edinburgh Sect. A 68 (1969), 145-157.

(14) A. P. Robertson and W. J. Robertson, Topological Vector Spaces (Cambridge University Press, Cambridge, 1964).

UNIVERSITY OF MASSACHUSETTS

AmHerst, MasSaCHUSETTS 01002

U.S.A. 